\title{
Effects of Soy Product Intake and Interleukin Genetic Polymorphisms on Early Gastric Cancer Risk in Korea: A Case-Control Study
}

Sarah Yang, $\mathrm{PhD}^{1,2}$
Yoon Park, $\mathrm{MPH}^{1}$
Jeonghee Lee, $\mathrm{MS}^{1}$
II Ju Choi, $\mathrm{MD}, \mathrm{PhD}^{3}$
Young Woo Kim, MD, $\mathrm{PhD}^{3}$
Keun Won Ryu, MD, $\mathrm{PhD}^{3}$
Joohon Sung, MD, $\mathrm{PhD}^{2}$
Jeongseon Kim, $\mathrm{PhD}^{1}$

${ }^{1}$ Molecular Epidemiology Branch, Division of Cancer Epidemiology and Prevention, Research Institute, National Cancer Center, Goyang, ${ }^{2}$ Complex Disease and Genome Epidemiology Branch, Department of Public Health, Graduate School of Public Health, Seoul National University, Seoul, ${ }^{3}$ Center for Gastric Cancer, National Cancer Center Hospital, National Cancer Center, Goyang, Korea

Correspondence: Jeongseon Kim, PhD Molecular Epidemiology Branch, Division of Cancer Epidemiology and Prevention, Research Institute, National Cancer Center, 323 Ilsan-ro, Ilsandong-gu, Goyang 10408, Korea Tel: $82-31-920-2570$

Fax: 82-31-920-2579

E-mail: jskim@ncc.re.kr

Received October 27, 2016

Accepted January 4, 2017

Published Online January 19, 2017

*Sarah Yang and Yoon Park contributed equally to this work.

\begin{abstract}
Purpose
The current study investigated whether the combined effects of soy intake and genetic polymorphisms of interleukin (IL) genes modify gastric cancer risk.
\end{abstract}

\begin{abstract}
Materials and Methods
A total of 377 cases and 754 controls of Korean origin were included in the analysis. Soy consumption was assessed using a semi-quantitative food frequency questionnaire. Seven variants of IL10 (rs1800871), IL2 (rs2069763 and rs2069762), IL13 (rs6596090 and rs20541), and IL4R (rs7205663 and rs1805010) were genetically analyzed. To analyze the combined effect of soy intake and genetic polymorphisms, a low-intake group and high-intake group of each type of soy were categorized based on the intake level of the control group. Interactions between soy products and these genetic variants were analyzed by a likelihood ratio test, in which a multiplicative interaction term was added to the logistic regression model.
\end{abstract}

\section{Results}

A higher intake of nonfermented soy products was associated with a reduced cancer risk (odds ratio [OR], 0.62; 95\% confidence interval [Cl], 0.43 to 0.90 ), and the reduced risk was only apparent in males (OR, $0.44 ; 95 \% \mathrm{Cl}, 0.27$ to 0.71 ). None of the IL genetic polymorphisms examined were independently associated with gastric cancer risk. Individuals with a minor allele of IL2 rs2069762 and a higher intake of nonfermented soy food had a decreased risk of gastric cancer (OR, $0.46 ; 95 \% \mathrm{Cl}, 0.31$ to 0.68 ) compared to those with a lower intake $\left(p_{\text {interaction }}=0.039\right)$.

\section{Conclusion}

Based on the genetic characteristics of the studied individuals, the interaction between IL2 rs2069762 and nonfermented soy intake may modify the risk of gastric cancer.

\section{Key words}

Stomach neoplasms, Soy foods, Interleukins, Genetic polymorphisms, Gene-environment interaction, Case-control studies, Korea

\section{Introduction}

Gastric cancer is the fifth most commonly diagnosed cancer worldwide [1]. The incidence rates of gastric cancer are the highest in East Asian countries, including Korea [2]. In Korea, gastric cancer is the second most common type of can- cer, although a gradual decline in incidence has been noted, with annual changes of $-0.6 \%$ occurring between 1999 and 2013. For Korean males, the incidence of gastric cancer is the highest among all types of cancer [3].

Gastric inflammation is a prerequisite for the development of gastric cancer in the multistage model of gastric carcinogenesis [4]. One of the main risk factors for gastric cancer is 
Helicobacter pylori infection, which has been classified by the International Agency for Research on Cancer as "carcinogenic to humans (Group 1)" [5]. H. pylori infection causes a chronic gastric inflammatory response and oxidative stress with a high chance of bacterial proliferation in the stomach that leads to diverse clinical outcomes in humans (e.g., gastritis, peptic ulcers, and gastric cancer) [6]. It has been recognized that gastric adenocarcinomas have frequently been found in areas of inflammation in which chronic $H$. pylori progresses over time [7]. However, clinical outcomes may differ depending on both genetic (intrinsic) and environmental (extrinsic) factors such as genetic variability of the $H$. pylori strain, genetic background of the infected host with different ethnic groups, and diversity of the diet or lifestyle $[6,8]$. Specifically, the inflammatory response can be induced by the virulence properties of $H$. pylori pathogenicity and by the genetic predisposition of inflammatory cytokines related to host immunity [9]. The cytokines encoded by the interleukin (IL) genes are thought to contribute to induction of the precancerous stage of gastric atrophy by regulating immune responses and gastric acid secretion and by inhibiting $H$. pylori infection [10]. Additionally, the dietary consumption of specific foods and their active constituents may impact the progression of gastric inflammation and carcinogenesis [11]. Among diverse foods consumed abundantly in Asian diets, soy products have been reported to have anti-inflammatory properties, and previous studies have confirmed that high soy consumption may reduce the risk of gastric cancer [12].

In this context, gastric cancer is believed to be affected by various factors, including genetic susceptibility, cultural diversity, ethnicity, sex, and other environmental influences, such as diets that differ among geographic regions [13]. The present study was conducted to investigate whether the combined effect of dietary soy consumption and inflammationrelated genetic polymorphisms (IL10, IL2, IL13, and IL4R) alters gastric cancer risk.

\section{Materials and Methods}

\section{Study participants and data collection}

The study subjects were recruited for a gastric cancer research project of the National Cancer Center (NCC), Korea between March 2011 and December 2014. The patients, who were diagnosed with early-stage gastric cancer three months prior to recruitment, were defined as the cases. Patients diagnosed with other cancers within 5 years, advanced gastric cancer, diabetes mellitus, severe systemic or mental disease, women who were pregnant or breastfeeding, and patients who stated that they had changed their dietary pattern due to illnesses were excluded. The controls of this study were recruited among individuals who visited the Center for Cancer Prevention and Detection at the same hospital as the beneficiaries of the National Health Insurance program, were in attendance to receive health examinations, and agreed to participate in the study. Individuals with a history of cancer, diabetes mellitus, gastric ulcers, and $H$. pylori treatment in the control group were excluded. Participants were asked to complete a set of self-administered surveys to obtain information regarding the demographics, medical history, lifestyle, and dietary intake habits. The frequency of cases and controls were matched for gender and 5-year age distributions. A total of 377 gastric cancer cases and 754 healthy controls for whom data on soy product intake and genetic characteristics were available were included in this study, and all of the participants were of Korean origin (Fig. 1). The infection status of $H$. pylori was evaluated using the rapid urease test (Pronto Dry, Medical Instruments Corp., Solothurn, Switzerland) and a histological/serological assessment. All of the participating subjects provided written informed consent, and the study protocol was approved by the Institutional Review Board of the National Cancer Center (NCCNCS 11-438).

\section{Dietary measurement}

Individuals were asked to complete a semi-quantitative food frequency questionnaire [14] consisting of 106 food items to obtain information regarding regular dietary intake habits from the past 12 months (participants were asked about the average frequency of intake and portion size of specific foods to assess their regular intake during the previous year using the validated food frequency questionnaire). The soy food items measured were legumes, tofu, soymilk, sprouts, and doenjang (Korean traditional fermented soybean paste and soybeans), and the intake amount of each item was calculated using CAN-PRO 4.0 (Computer Aided Nutritional Analysis Program, The Korean Nutrition Society, Seoul, Korea). The isoflavone intake level from the five types of soy food consumption was estimated using the Korean Isoflavone database [15].

\section{Genotype measurement}

Genomic DNA was extracted using peripheral blood leukocytes isolated from whole-blood samples obtained from the participants. The Affymetrix Axiom Exom 319 Array (Affymetrix Inc., Santa Clara, CA) platform, including 318,983 variants, was used for genotyping. Genetic markers with deviation from Hardy-Weinberg equilibrium p-values $<1 \times 10^{-6}$, a minor allele frequency (MAF) $<0.01$, and a low 


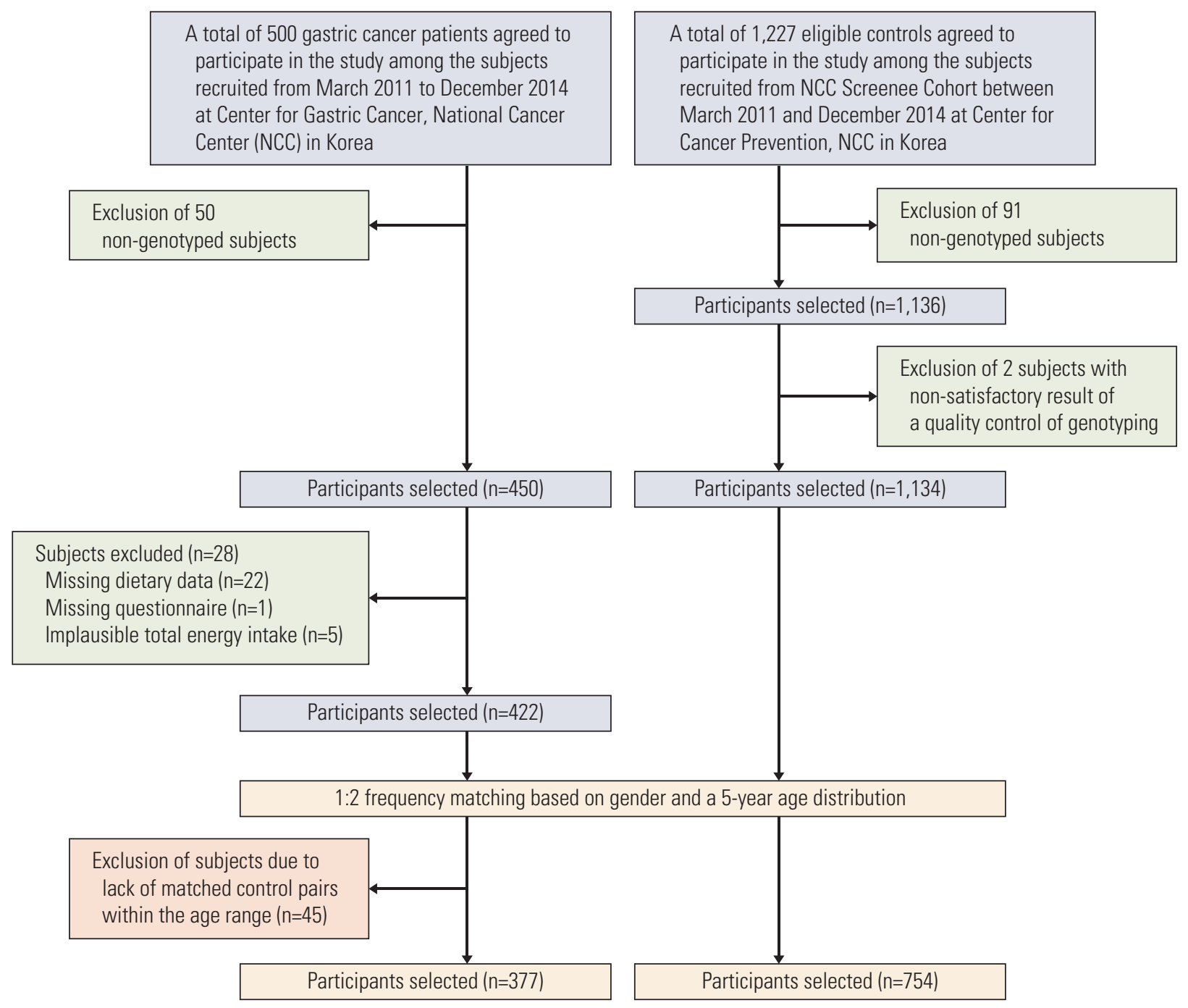

Fig. 1. Flowchart for the selection of study subjects in the study.

call rate $(<90 \%)$ were discarded. The genotype imputation was performed using the Asian population $(n=504)$ of 1000 Genome haplotypes phase III integrated variant set release GRch37/hg19 (http://www.1000genomes.org/) as a reference panel. Phasing was performed using SHAPIT (v2.r837), and single-nucleotide polymorphism (SNP) imputation was completed using IMPUTE2 (2.3.2). The same quality control criteria were applied after filtering for an INFO score over 0.6. A total of seven variants of IL10, IL2, IL13, and IL4R (rs1800871, rs2069763, rs2069762, rs6596090, rs20541, rs7205663, and rs1805010, respectively) were selected. The MAFs of all variants were over 0.15 , and imputed variants (rs2069762, rs6596090, rs7205663) had an INFO score above 0.95. Detailed information regarding the variants analyzed in this study can be found in S1 Table.

\section{Statistical analyses}

The demographics and general characteristics of the study participants were compared between the case and control subjects using the Student's t test for continuous variables and the chi-square test for categorical variables. The dietary variables were adjusted for total daily calorie intake for statistical analysis using the regression residual method [16]. The legume, tofu, soymilk, and sprout intakes were combined to represent nonfermented soy products, and the total soy product intake was calculated by adding all five types of soy foods. Based on the distribution of the control samples, the food intakes were categorized into tertiles for further analysis. Odds ratios (OR) and 95\% confidence intervals (CI) of gastric cancer risk were calculated for each soy food group 
Table 1. General characteristics of study participants, stratified by cancer status

\begin{tabular}{|c|c|c|c|}
\hline Characteristic & Case $(n=377)$ & Control $(\mathrm{n}=754)$ & p-value ${ }^{a)}$ \\
\hline Age (yr) & $53.90 \pm 9.19$ & $53.83 \pm 9.05$ & 0.900 \\
\hline \multicolumn{4}{|l|}{ Sex } \\
\hline Male & $246(65.25)$ & $492(65.25)$ & $>0.999$ \\
\hline Female & $131(34.75)$ & $262(34.75)$ & \\
\hline \multicolumn{4}{|l|}{ Histological type } \\
\hline Intestinal & $144(38.20)$ & & \\
\hline Diffuse & $151(40.05)$ & & \\
\hline Mixed & $53(14.06)$ & & \\
\hline Missing & $29(7.69)$ & & \\
\hline \multicolumn{4}{|l|}{ Alcohol drinking status } \\
\hline Non-drinker & $111(29.44)$ & $218(28.91)$ & 0.358 \\
\hline Ex-drinker & $38(10.08)$ & $58(7.69)$ & \\
\hline Current drinker & $228(60.48)$ & $478(63.40)$ & \\
\hline \multicolumn{4}{|l|}{ Smoking status } \\
\hline Non-smoker & $152(40.32)$ & $344(45.62)$ & 0.001 \\
\hline Ex-smoker & $110(29.18)$ & $256(33.95)$ & \\
\hline Current smoker & $115(30.50)$ & $154(20.42)$ & \\
\hline \multicolumn{4}{|l|}{ Education } \\
\hline Elementary & $54(14.36)$ & $43(5.97)$ & $<0.001$ \\
\hline Middle-high & $237(63.03)$ & $295(40.97)$ & \\
\hline University & $85(22.61)$ & $382(53.06)$ & \\
\hline \multicolumn{4}{|l|}{ Regular exercise } \\
\hline Yes & $136(36.07)$ & $416(55.39)$ & $<0.001$ \\
\hline No & $241(63.93)$ & $335(44.61)$ & \\
\hline \multicolumn{4}{|l|}{ Helicobacter pylori infection } \\
\hline Negative & $28(7.43)$ & $288(38.20)$ & $<0.001$ \\
\hline Positive & $349(92.57)$ & $466(61.80)$ & \\
\hline \multicolumn{4}{|l|}{ Dietary intake ${ }^{\text {b) }}$} \\
\hline Isoflavone (mg/ day) & $13.82 \pm 9.74$ & $15.56 \pm 12.22$ & 0.009 \\
\hline Legumes (g/day) & $3.99 \pm 7.07$ & $5.80 \pm 10.97$ & 0.001 \\
\hline Tofu (g/day) & $36.10 \pm 30.73$ & $38.45 \pm 33.30$ & 0.252 \\
\hline Soymilk (g/day) & $122.76 \pm 1,210.09$ & $337.6 \pm 5,904.90$ & 0.338 \\
\hline Sprouts (g/day) & $14.08 \pm 16.18$ & $16.49 \pm 19.92$ & 0.029 \\
\hline Soybean paste (g/ day) & $6.32 \pm 7.81$ & $5.99 \pm 7.21$ & 0.482 \\
\hline Total soy product (g/day) & $71.75 \pm 51.61$ & $82.88 \pm 77.25$ & 0.005 \\
\hline
\end{tabular}

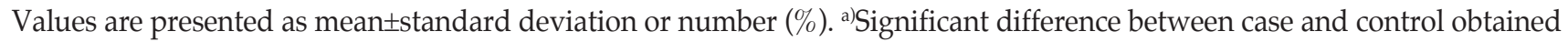

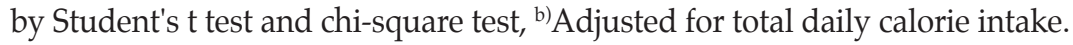

and for isoflavone using multiple logistic regression, with the lowest tertile as a reference group. The estimates of ORs and $95 \%$ CIs were adjusted for education level, alcohol consumption status, smoking status, H. pylori infection, and engagement in regular exercise. Each genetic variant's risk of gastric cancer was assessed under the dominant model. To analyze the combined effects of soy food intake and genetic polymorphism, a low-intake group and a high-intake group for each type of soy food were categorized according to the median intake level of the control group. OR and the
95\% CI of each of the stratified groups were estimated, and the interactions between soy products and SNPs were analyzed by a likelihood ratio test, where the multiplicative interaction term of the genetic polymorphism and soy food intake was added to the logistic regression model. The cases and their individually matched controls were stratified by histological type and the interactions between nonfermented soy product intake and IL polymorphisms were further analyzed. All of the statistical analysis was performed using SAS ver. 9.3 (SAS Institute Inc., Cary, NC). 


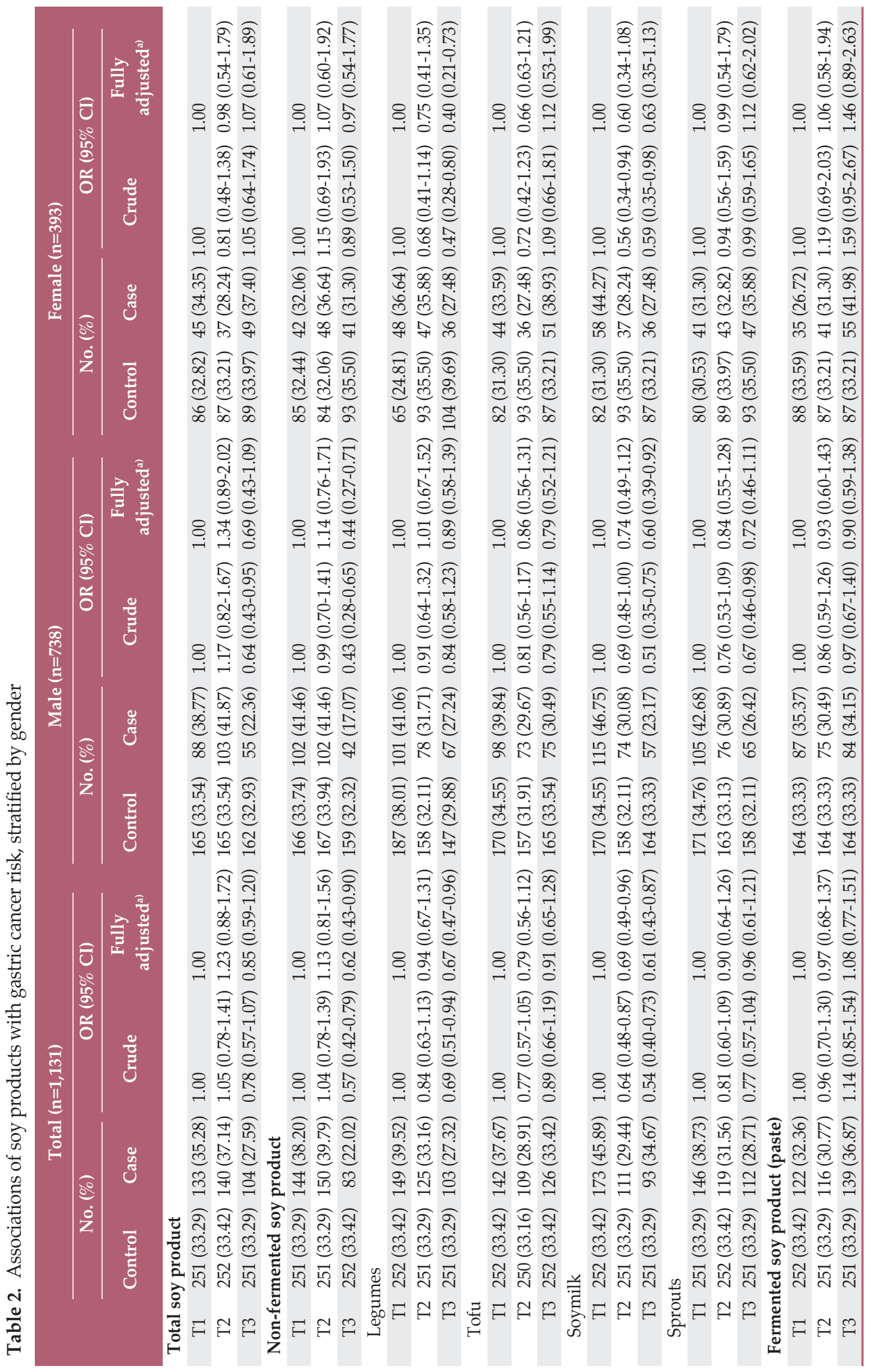




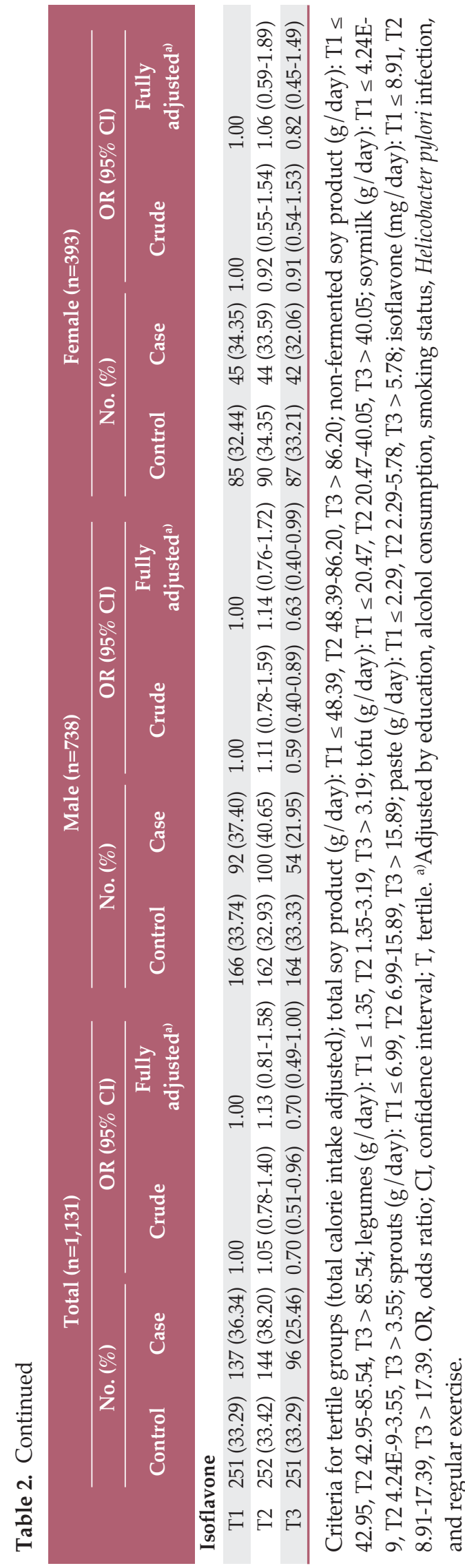

\section{Results}

Daily intakes (g/day) of total soy products, legumes, and sprouts and isoflavone levels ( $\mathrm{mg} /$ day) were higher in the control group. There were no age or gender differences between the cases and controls because the participants were matched for frequency. The cases had a higher proportion of $H$. pylori infection, nonregular physical exercisers, and current smokers and a lower proportion of college graduates (Table 1).

Male individuals with the highest tertile total soy product intake had a lower risk for gastric cancer than the lowest tertile group (OR, 0.64; 95\% CI, 0.43 to 0.95 ) in the crude model, but the association was null in the fully adjusted model. A higher intake of nonfermented soy products was associated with reduced cancer risk in the total population (OR, 0.62; $95 \%$ CI, 0.42 to 0.89 ), and the reduced risk was only apparent in males (OR, 0.44; 95\% CI, 0.27 to 0.71). However, a higher intake of legumes, which is a subgroup of nonfermented soy, was associated with reduced gastric cancer risk in the total population (OR, $0.67 ; 95 \% \mathrm{CI}, 0.47$ to 0.96$)$ and in females (OR, 0.40; 95\% CI, 0.21 to 0.73 ). Soymilk intake was also inversely associated with gastric cancer risk, but a protective effect of isoflavone was only observed in males (Table 2). None of the interleukin genetic polymorphisms examined in this study were independently associated with all-type gastric cancer risk (Table 3); however, increased diffuse-type gastric cancer risk was observed for IL2 rs2069763, and IL13 rs6596090 was associated with mixed-type gastric cancer (S2 Table).

A higher intake of nonfermented soy food among individuals with the $C$ allele of rs2069762 displayed a significant association (OR, $0.46 ; 95 \% \mathrm{CI}, 0.31$ to 0.68$)$, whereas OR was estimated as 0.84 (95\% CI, 0.54 to 1.29) among AA homozygotes. The interacting effects for IL2 rs2069762 and nonfermented soy product intake were significant $\left(p_{\text {interaction }}=0.039\right.$ ). Several protective effects of nonfermented soy intake were observed for groups stratified by other remaining interleukin SNPs, but no significant interaction was observed. When the effect was assessed according to histological type of cancer, stronger evidence of an interaction between nonfermented soy intake and rs2069762 was observed for intestinal-type gastric cancer ( $p_{\text {interaction }}=0.001$ ). A possible interaction between nonfermented soy food and IL10 rs1800871 was also observed for intestinal-type gastric cancer ( $p_{\text {interaction }}=0.033$ ). No significant interaction was found for diffuse-type, and there was evidence of a marginal interacting effect between nonfermented soy products and IL13 rs20541 (pinteraction= 0.050) for mixed-type gastric cancer (Table 4). No apparent interaction was observed for total soy, isoflavone, and fermented paste when stratified by IL genetic variants (S3 Table). 
Table 3. Associations of interleukin genetic polymorphisms (dominant model) with gastric cancer risk

\begin{tabular}{|c|c|c|c|c|c|c|}
\hline \multirow{2}{*}{ Gene } & \multirow{2}{*}{ rs No. } & & \multicolumn{2}{|c|}{ No. $(\%)$} & \multicolumn{2}{|c|}{ OR $(95 \% \mathrm{CI})$} \\
\hline & & & Control & Case & Crude & Fully adjusted \\
\hline \multirow[t]{2}{*}{ IL10 } & rs1800871 & $\mathrm{AA}$ & $368(48.81)$ & $173(45.89)$ & 1 & 1 \\
\hline & & G+ & 386 (51.19) & 204 (54.11) & $1.12(0.88-1.44)$ & $1.06(0.80-1.41)$ \\
\hline \multirow[t]{4}{*}{ IL2 } & rs2069763 & $\mathrm{AA}$ & $226(29.97)$ & $1111(29.44)$ & 1 & 1 \\
\hline & & $\mathrm{C}+$ & $528(70.03)$ & $266(70.56)$ & $1.03(0.78-1.35)$ & $1.19(0.88-1.62)$ \\
\hline & rs2069762 & $\mathrm{AA}$ & 358 (47.73) & 165 (43.77) & 1 & 1 \\
\hline & & $\mathrm{C}+$ & $392(52.27)$ & $212(56.23)$ & $1.17(0.92-1.51)$ & $1.23(0.93-1.64)$ \\
\hline \multirow[t]{4}{*}{ IL13 } & rs6596090 & GG & $517(70.24)$ & 255 (69.29) & 1 & 1 \\
\hline & & $\mathrm{A}+$ & 219 (29.76) & $113(30.71)$ & $1.05(0.80-1.37)$ & $1.04(0.77-1.43)$ \\
\hline & rs20541 & GG & $366(48.54)$ & $185(49.07)$ & 1 & 1 \\
\hline & & $\mathrm{A}+$ & $388(51.46)$ & $192(50.93)$ & $0.98(0.76-1.25)$ & $0.86(0.65-1.14)$ \\
\hline \multirow[t]{4}{*}{ IL4R } & rs7205663 & TT & 259 (34.35) & $126(33.42)$ & 1 & 1 \\
\hline & & $\mathrm{C}+$ & 495 (65.65) & $251(66.58)$ & $1.04(0.80-1.35)$ & $0.99(0.73-1.33)$ \\
\hline & rs1805010 & GG & $253(33.55)$ & $126(33.42)$ & 1 & 1 \\
\hline & & $\mathrm{A}+$ & $501(66.45)$ & $251(66.58)$ & $1.01(0.77-1.31)$ & $0.95(0.70-1.28)$ \\
\hline
\end{tabular}

$\mathrm{OR}$, odds ratio; $\mathrm{CI}$, confidence interval. a)Adjusted by education, alcohol consumption, smoking status, Helicobacter pylori infection, and regular exercise.

The association between soy product intake, IL genetic polymorphism, and gastric cancer risk was further analyzed after stratification by H. pylori infection status (S4 TableS6 Table). For the association with each type of soy product, only the H. pylori positive groups showed a protective association with nonfermented soy products, legumes, soymilk, and isoflavone, which is consistent with the association results for the total study population. However, uninfected individuals did not display any significant association after adjustment for covariates. Additionally, when the effect of IL genetic polymorphisms was analyzed, IL2 rs2067962 had a significant relationship with the disease among members of the H. pylori infection-negative group (OR, 2.88; 95\% CI, 1.14 to 7.26$)$.

\section{Discussion}

Our findings demonstrate that the dietary intake of nonfermented soy food items was associated with a decrease in gastric cancer risk, whereas there was no independent association between IL genetic polymorphisms and intestinaltype gastric cancer in contrast to the associations observed for diffuse-type and mixed-type gastric cancer risk. Among individuals with higher intake of nonfermented soy products, only those carrying the minor allele C of IL2 rs2069762 showed a protective effect of soy against gastric cancer risk compared to individuals with different genetic characteristics. This trend was more apparent for intestinal-type gastric cancer risk than diffuse-type.

Gastric cancer is a common diet-related cancers with an etiology that can be explained based on differences in environmental risk factors. Diet has been regarded as a complex environmental factor, with diversity in the common diets of each culture due to different major food sources, cooking or storing methods, and recipes [11]. Soy products are abundantly used in Korean dishes, and soybeans and soybeanderived foods are a rich source of bioactive phytochemicals with anti-inflammatory or anti-cancer activity in different types of carcinogenesis [17]. Our study demonstrated that consumption of nonfermented soy, including legumes and soymilk, was associated with a significant decrease in gastric cancer risk. However, there was no evidence of an association between gastric cancer risk and the intake of fermented soy products, such as soybean paste, which is frequently used in Korean recipes and known to have a high salt content. According to a comprehensive literature review published by the World Cancer Research Fund and the American Institute for Cancer Research [18], salted and salty foods are "probable" factors that increase the risk of gastric cancer, and soy products are classified as "limited suggestive" foods to decrease gastric cancer risk. Recommendations suggest that the dietary intake of nonfermented soy products may provide greater preventive effects against gastric cancer than the 


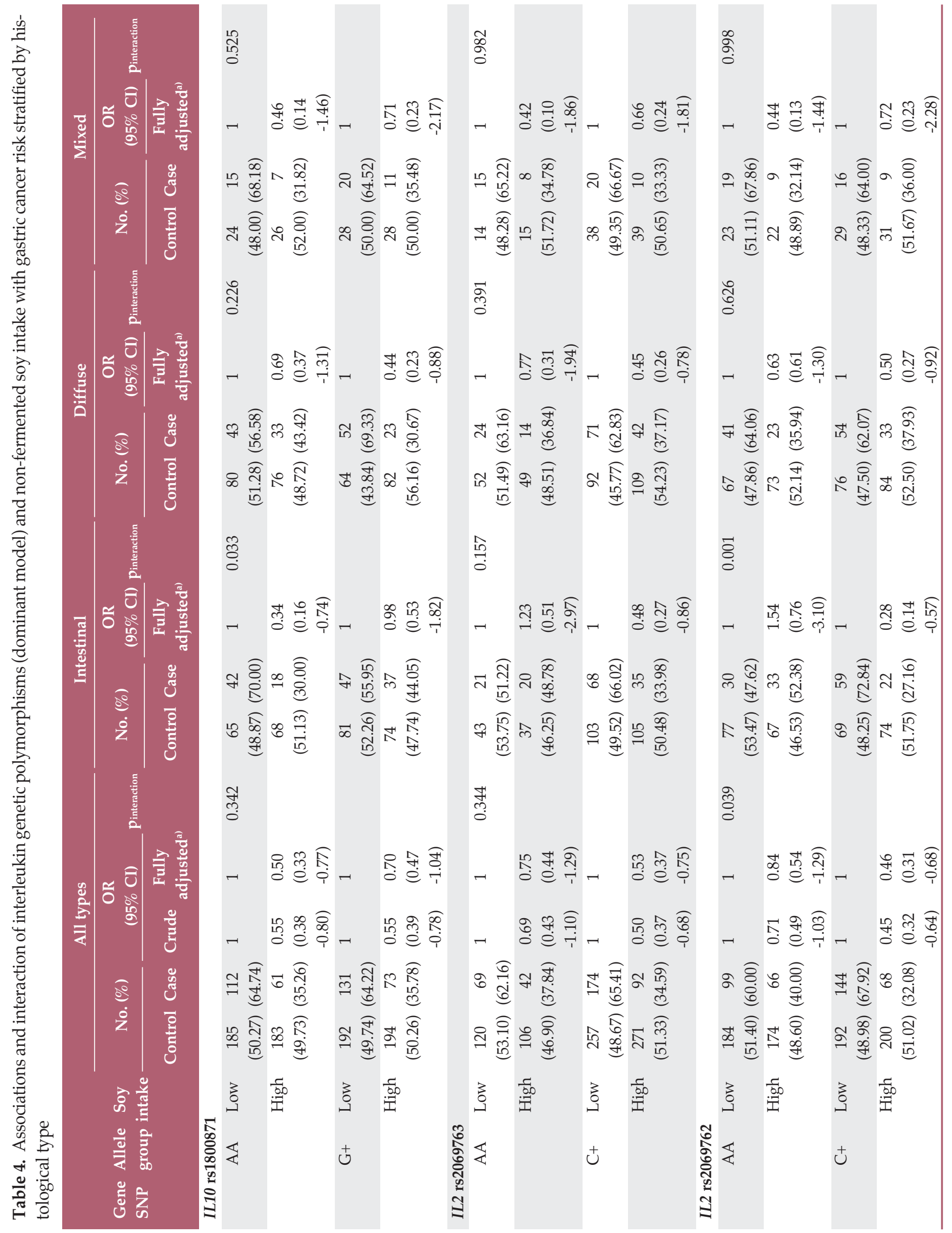




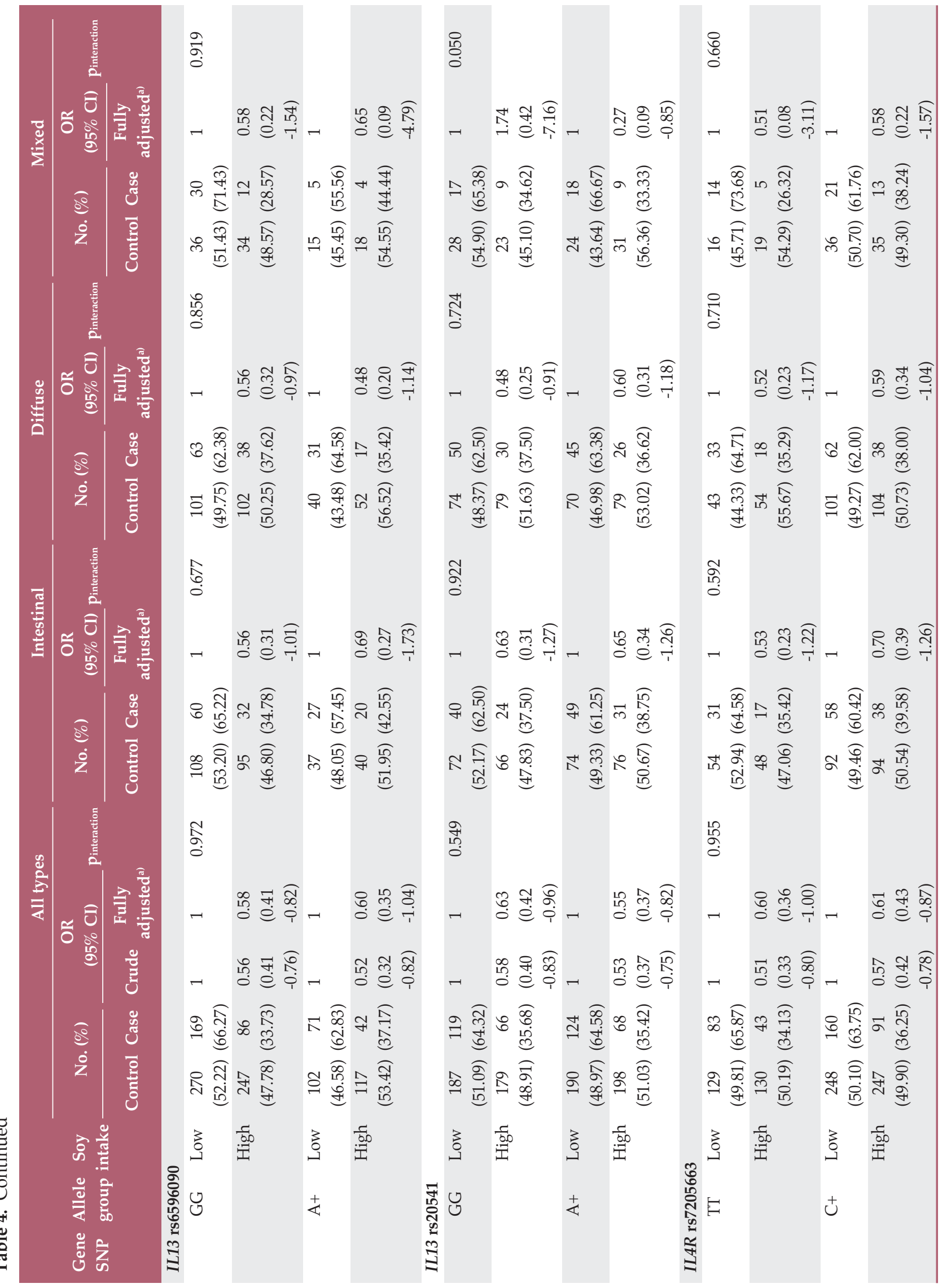


(a)

政的

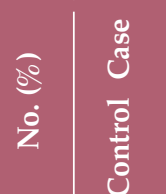

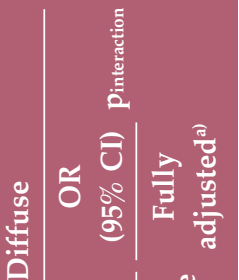

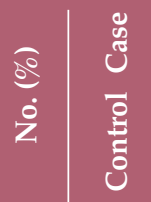

|

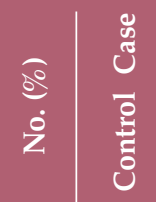

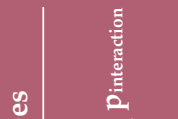

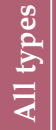

o็

हुँ

वें

نี

نَّ
ஸి

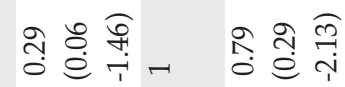

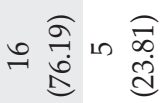

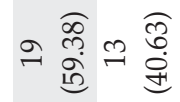

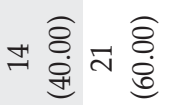

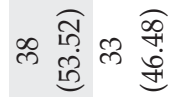

సิ

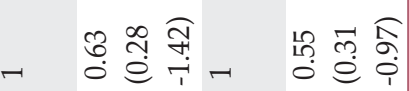

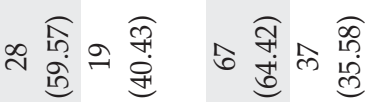

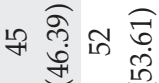

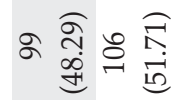

ণิ

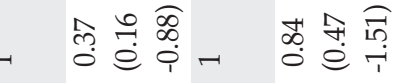

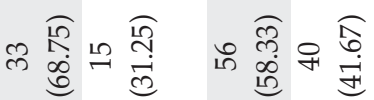

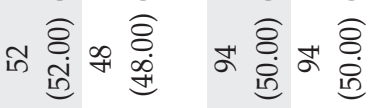

‡̊ำ

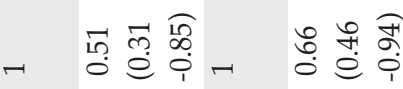

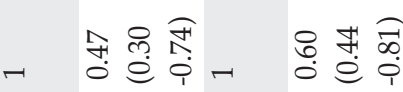

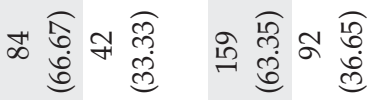

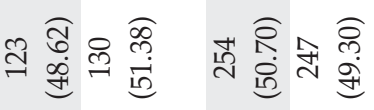

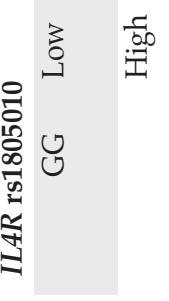

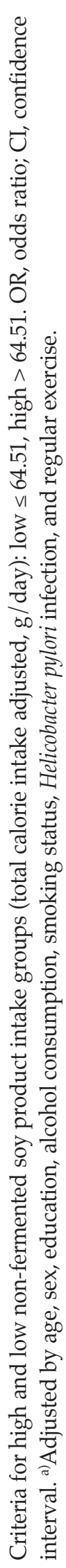


consumption of fermented soy products that include high levels of salt, such as preserved vegetables and condiments (e.g., Kimchi, pickles, soy sauce, doenjang) according to traditional Korean recipes. Stratification of our results by $H$. pylori infection status suggest that the anti-inflammatory and anti-oxidative effects of soy intake (e.g., nonfermented soy product, legumes, soymilk, and isoflavone) are helpful for individuals who are already susceptible to chronic inflammation or gastritis due to $H$. pylori infection. We hypothesized that dietary nonfermented soy intake may reduce the risk of gastric cancer by modulating immune parameters in favor of anti-cancer immune responses in humans $[4,19]$. Soy is well known as a beneficial food containing phytochemicals (e.g., isoflavone) that have anti-inflammatory and anti-oxidative effects. Phytochemicals generated in plants may overcome inflammation and infection by immune modulation through its components [20]. Specifically, soy is known to potentiate immunological functions of lymphocyte proliferation, cellular and humoral immune responses, thymocyte differentiation, and tumor immunity [21]. We expected that soy isoflavone would benefit individuals by inhibiting the progression of inflammation and gastric carcinogenesis through the anti-carcinogenic properties of phytochemicals (e.g., phytoestrogens) [22]. Our findings demonstrate that the intake of soy isoflavone is associated with a decrease in gastric cancer risk, particularly in males, whereas there was no association of risk in females. Moreover, reduced gastric cancer risk was shown in females consuming legumes, although no association was observed in males. Further studies are required to support the role of soy phytochemicals as phytoestrogens in association with gastric cancer and to reveal the related biological mechanism, gender difference, and genetic susceptibility.

Genetic variants encoding cytokines may influence an individual's inflammatory response and health or clinical outcomes [4]. Genetic polymorphisms of cytokines have been associated with variations in the level of transcription and expression of cytokines that can exert activities in human diseases [23]. Many candidate gene studies have been conducted, and the results have indicated that polymorphisms in cytokine genes influence the susceptibility to gastric cancer and clinical outcomes (e.g., the course, symptoms, and treatments related to gastric cancer) [23,24]. Cytokine production generated by immune and inflammatory cells is one of the major tumor-promoting mechanisms. Cytokines can have either pro- or anti-inflammatory activity and may be involved in immunomodulatory activity depending on the microenvironment [24]. The immunomodulatory response is served by the balance between anti-inflammatory cytokines (e.g., IL4, IL10, and IL13) and pro-inflammatory cytokines (e.g., IL2) [25]. With regard to gastric cancer, cytokine polymorphisms of the host are the most studied risk factor and result in an increased risk of gastric cancer by decreasing the anti-inflammatory host reaction or increasing the pro-inflammatory response [26]. We hypothesized that the risk for gastric cancer may be modified by IL genetic variations, which result in differences in immunomodulatory activity among individuals. Independent risk associations between IL polymorphisms and intestinal-type gastric cancer were not observed in this study, but an association between IL2 rs2069763 and diffuse-type gastric cancer risk was observed. Different effects of IL genetic polymorphisms for different histological types of gastric cancer are anticipated since the etiology of cancer development is diverse [7,27]. Diffuse-type gastric cancer is more closely associated with genetic susceptibility, whereas environmental factors, such as lifestyle and dietary patterns, reportedly have a greater influence on intestinal-type gastric cancer [27,28]. In addition, the genetic susceptibility to immune responses may be a strong risk factor for the development of gastric cancer among individuals without $H$. pylori infection.

A suggestive interaction was detected between nonfermented soy intake and IL2 polymorphism (rs2069762) for gastric cancer risk, especially for intestinal-type. The IL2 gene is an important cytokine family member that plays a critical role in carcinogenesis through the proliferation of activated $\mathrm{T}$ lymphocytes and participates in termination of the lymphocyte response by inducing suppressive T cells [29]. The IL2 gene polymorphism, which is associated with an increased risk of gastric atrophy induced by $H$. pylori infection, might predispose individuals to gastric cancer [10]. Previous studies have investigated the association between rs2069762 polymorphism and cancer risk, but the results remain controversial $[10,29]$. We found a suggestive interactive effect between by rs2069762 and nonfermented soy consumption on intestinal-type gastric cancer risk, which suggests a possible interactive role between soy phytochemicals and the pro-inflammatory properties of IL2 cytokines. No previous epidemiological studies have examined the effects of the interaction between soybean product intake and IL2 genetic variants on modification of gastric cancer risk, which is one of the strengths of this study. rs1800871 of IL10 also showed a suggestive interacting effect with nonfermented soy product intake on intestinal-type gastric cancer risk, which confirms the results of a previous study that examined the interactive effect between soy and IL10 [30] and suggested a possible interaction between the anti-inflammatory properties of cytokines and soybean products. Further epidemiological and experimental studies are required to support this unclear interactive effect.

It should be noted that this study has several strengths and limitations. The dietary data used in the analysis were more robust than those employed in previous studies focusing on the interactive effect between soy consumption and 
inflammatory genes with regard to gastric cancer risk. In addition, the dietary data included various types of soy products that were all quantitatively comparable (e.g., $\mathrm{g} /$ day), whereas similar studies utilized dietary data that represented only the frequency of intake (e.g., times per week). Although the dietary data including detailed soy items strengthened this study, recall or reporting bias may be present. Thus, this study was limited with regard to representing changes in dietary or lifestyle habits due to illness (whether the change preceded of followed illness). Additionally, our statistical p-values were not significant if a strict multiple comparison adjustment was applied. Nonetheless, our findings imply a suggestive interaction between soy intake and IL2 genotypes.

In conclusion, our study of a Korean population suggested that the interaction between IL2 rs2069762 and nonfermented soy intake may modify the risk of gastric cancer. The findings of this study suggest that an interaction between a variant of the IL2 gene and phytochemicals in nonfermented soy products has a preventative effect on gastric cancer, and that this observation may support the promotion of dietary intervention against gastric cancer on the basis of potential geneenvironment interactions. Further studies are required to replicate the results and to clarify the corresponding biological mechanisms involved in gastric carcinogenesis.

\section{Electronic Supplementary Material}

Supplementary materials are available at Cancer Research and Treatment website (http:// www.e-crt.org).

\section{Conflicts of Interest}

Conflict of interest relevant to this article was not reported.

\section{Acknowledgments}

This work was supported by grants from the National Cancer Center of Korea (NCC1410260 and NCC1510040).

\section{References}

1. International Agency for Research on Cancer. Globocan 2012: estimated cancer incidence, mortality, and prevalence worldwide in 2012 [Internet]. Lyon: International Agency for Research on Cancer; 2015 [cited 2016 Jul 11]. Available from: http://globocan.iarc.fr/Default.aspx.

2. Varghese C, Carlos MC, Shin HR. Cancer burden and control in the Western Pacific region: challenges and opportunities. Ann Glob Health. 2014;80:358-69.

3. Oh CM, Won YJ, Jung KW, Kong HJ, Cho H, Lee JK, et al. Cancer statistics in Korea: incidence, mortality, survival, and prevalence in 2013. Cancer Res Treat. 2016;48:436-50.

4. Yang J, Hu Z, Xu Y, Shen J, Niu J, Hu X, et al. Interleukin-1B gene promoter variants are associated with an increased risk of gastric cancer in a Chinese population. Cancer Lett. 2004; 215:191-8.

5. International Agency for Research on Cancer. IARC monographs on the evaluation of carcinogenic risks to humans. Schistosomes, liver flukes and Helicobacter pylori. Lyon: IARC Press; 1994.

6. Zaidi SF. Helicobacter pylori associated Asian enigma: does diet deserve distinction? World J Gastrointest Oncol. 2016;8:341-50.

7. Fox JG, Wang TC. Inflammation, atrophy, and gastric cancer. J Clin Invest. 2007;117:60-9.

8. Garte S. Individual susceptibility and gene-environment interaction. In: Wild CP, Vineis P, Garte S, editors. Molecular epidemiology of chronic diseases. West Sussex: John Wiley \& Sons, Ltd.; 2008. p. 55-69.
9. Katoh M. Dysregulation of stem cell signaling network due to germline mutation, SNP, Helicobacter pylori infection, epigenetic change and genetic alteration in gastric cancer. Cancer Biol Ther. 2007;6:832-9.

10. Togawa S, Joh T, Itoh M, Katsuda N, Ito H, Matsuo K, et al. Interleukin-2 gene polymorphisms associated with increased risk of gastric atrophy from Helicobacter pylori infection. Helicobacter. 2005;10:172-8.

11. Tollefsbol TO. Dietary epigenetics in cancer and aging. Cancer Res Treat. 2014;159:257-67.

12. Woo HD, Park S, Oh K, Kim HJ, Shin HR, Moon HK, et al. Diet and cancer risk in the Korean population: a meta-analysis. Asian Pac J Cancer Prev. 2014;15:8509-19.

13. Milne AN, Carneiro F, O'Morain C, Offerhaus GJ. Nature meets nurture: molecular genetics of gastric cancer. Hum Genet. 2009;126:615-28.

14. Ahn Y, Kwon E, Shim JE, Park MK, Joo Y, Kimm K, et al. Validation and reproducibility of food frequency questionnaire for Korean genome epidemiologic study. Eur J Clin Nutr. 2007;61:1435-41.

15. Park MK, Song Y, Joung H, Li SJ, Paik HY. Establishment of an isoflavone database for usual Korean foods and evaluation of isoflavone intake among Korean children. Asia Pac J Clin Nutr. 2007;16:129-39.

16. Willett W. Implications of total energy intake for epidemiologic analyses. In: Willett W, editor. Nutritional epidemiology. 3rd ed. New York: Oxford University Press; 2013. p. 247-71.

17. Lesinski GB, Reville PK, Mace TA, Young GS, Ahn-Jarvis J, 
Thomas-Ahner J, et al. Consumption of soy isoflavone enriched bread in men with prostate cancer is associated with reduced proinflammatory cytokines and immunosuppressive cells. Cancer Prev Res (Phila). 2015;8:1036-44.

18. World Cancer Research Fund; American Institute for Cancer Research. Food, nutrition, physical activity, and the prevention of cancer: a global perspective. 2nd ed. Washington, DC: World Cancer Research Fund, American Institute for Cancer Research; 2007. p. 265.

19. Wu J, Lu Y, Ding YB, Ke Q, Hu ZB, Yan ZG, et al. Promoter polymorphisms of IL2, IL4, and risk of gastric cancer in a highrisk Chinese population. Mol Carcinog. 2009;48:626-32.

20. Tezuka H, Imai S. Immunomodulatory effects of soybeans and processed soy food compounds. Recent Pat Food Nutr Agric. 2015;7:92-9.

21. Handayani R, Rice L, Cui Y, Medrano TA, Samedi VG, Baker $\mathrm{HV}$, et al. Soy isoflavones alter expression of genes associated with cancer progression, including interleukin-8, in androgenindependent PC-3 human prostate cancer cells. J Nutr. 2006; 136:75-82.

22. Ko KP, Park SK, Park B, Yang JJ, Cho LY, Kang C, et al. Isoflavones from phytoestrogens and gastric cancer risk: a nested case-control study within the Korean Multicenter Cancer Cohort. Cancer Epidemiol Biomarkers Prev. 2010;19: 1292-300.

23. Dong LM, Potter JD, White E, Ulrich CM, Cardon LR, Peters
U. Genetic susceptibility to cancer: the role of polymorphisms in candidate genes. JAMA. 2008;299:2423-36.

24. Seruga B, Zhang H, Bernstein LJ, Tannock IF. Cytokines and their relationship to the symptoms and outcome of cancer. Nat Rev Cancer. 2008;8:887-99.

25. Opal SM, DePalo VA. Anti-inflammatory cytokines. Chest. 2000;117:1162-72.

26. Burada F, Angelescu C, Mitrut P, Ciurea T, Cruce M, Saftoiu $\mathrm{A}$, et al. Interleukin-4 receptor $-3223 \mathrm{C} \rightarrow \mathrm{T}$ polymorphism is associated with increased gastric adenocarcinoma risk. Can J Gastroenterol. 2012;26:532-6.

27. Hu B, El Hajj N, Sittler S, Lammert N, Barnes R, Meloni-Ehrig A. Gastric cancer: classification, histology and application of molecular pathology. J Gastrointest Oncol. 2012;3:251-61.

28. Epplein M, Nomura AM, Hankin JH, Blaser MJ, Perez-Perez G, Stemmermann GN, et al. Association of Helicobacter pylori infection and diet on the risk of gastric cancer: a case-control study in Hawaii. Cancer Causes Control. 2008;19:869-77.

29. Wang Y, Shu Y, Jiang H, Sun B, Ma Z, Tang W. Lack of association between interleukin-2 (IL-2) gene rs2069762 polymorphism and cancer risk: a meta-analysis. Int J Clin Exp Med. 2015;8:12557-65.

30. Ko KP, Park SK, Cho LY, Gwack J, Yang JJ, Shin A, et al. Soybean product intake modifies the association between interleukin-10 genetic polymorphisms and gastric cancer risk. J Nutr. 2009;139:1008-12. 